\title{
New Production Mechanism for Heavy Neutrinos at the LHC
}

\author{
P. S. Bhupal Dev, ${ }^{1}$ Apostolos Pilaftsis, ${ }^{1,2}$ and Un-ki Yang ${ }^{3}$ \\ ${ }^{1}$ Consortium for Fundamental Physics, School of Physics and Astronomy, University of Manchester, \\ Manchester M13 9PL, United Kingdom \\ ${ }^{2}$ CERN, Department of Physics, Theory Division, CH-1211 Geneva 23, Switzerland \\ ${ }^{3}$ Department of Physics and Astronomy, Seoul National University, Seoul 151-747, Korea
}

(Received 15 August 2013; revised manuscript received 5 November 2013; published 24 February 2014)

\begin{abstract}
We study a new production mechanism for heavy neutrinos at the LHC, which dominates over the usually considered $s$-channel $W$-exchange diagram for heavy-neutrino masses larger than 100-200 GeV. The new mechanism is infrared enhanced by $t$-channel $W \gamma$-fusion processes. This has important implications for experimental tests of the seesaw mechanism of neutrino masses and, in particular, for the ongoing heavy neutrino searches at the LHC. We find that the direct collider limits on the light-to-heavy neutrino mixing can be significantly improved when this new production channel is properly taken into account. The scope of this new mechanism can be equally well extended to other exotic searches at the LHC.
\end{abstract}

The discovery of nonzero neutrino masses and mixing from neutrino oscillation data provides the first (and so far only) conclusive experimental evidence of the existence of new physics beyond the Standard Model (SM). A simple paradigm for understanding the smallness of neutrino masses in a natural way is the seesaw mechanism [1]. Its simplest realization [2] (known as the type-I seesaw) requires the existence of a set of heavy SM-singlet Majorana fermions $N$, which break the $(B-L)$ symmetry of the theory by two units. The seesaw scale is synonymous with the typical Majorana mass $M_{N}$ of these heavy neutrinos, whose origin must be connected with some new physics [1]. In the flavor basis $\left\{\left(\nu_{L}\right)^{C}, N\right\}$, the seesaw mass matrix has the following general structure $[2,3]$ :

$$
\mathcal{M}_{\nu}=\left(\begin{array}{cc}
0 & M_{D} \\
M_{D}^{\top} & M_{N}
\end{array}\right)
$$

where $M_{D}$ is the Dirac mass term that mixes the light $\left(\nu_{L}\right)$ and heavy $(N)$ states. In the usual seesaw approximation, $\|\xi\| \ll 1$, where $\xi \equiv M_{D} M_{N}^{-1}$ and $\|\xi\| \equiv \sqrt{\operatorname{Tr}\left(\xi^{\dagger} \xi\right)}$, this leads to the observed light neutrino mass matrix of the form

$$
M_{\nu} \simeq-M_{D} M_{N}^{-1} M_{D}^{\top}
$$

and to the light-to-heavy neutrino mixing of order $\xi$ [3]. We note that the smallness of $M_{\nu}$ could be attributed to a very high value for $M_{N}$, or to a particular flavor structure in (2), or both. Without specifying the details of the model, we generically call this minimal realization the "SM seesaw."

As mentioned above, there are two key aspects of the seesaw mechanism that can be probed experimentally: the Majorana mass $M_{N}$ of the heavy neutrinos and the mixing $\xi$ between the heavy and light neutrinos. The Majorana nature of the light and heavy neutrinos can in principle be tested via neutrinoless double beta decay $(0 \nu \beta \beta)$ [4]. However, this does not necessarily probe the mixing $\xi$ whose effects may be sub-dominant, compared to purely left-(or right-)handed contributions to the $0 \nu \beta \beta$ process. Alternatively, a non-negligible value for $\xi$ could be inferred from nonunitarity of the light neutrino mixing matrix [5], in neutrino oscillation data, as well as in observables for lepton flavor violation (LFV) [6]. However, these lowenergy observables by themselves do not prove the Majorana nature of heavy neutrinos since models with pseudo-Dirac heavy neutrinos can also yield large nonunitarity and LFV effects [7].

In the SM seesaw, the Majorana nature of possible electroweak-scale heavy neutrinos as well as their mixing with the light neutrinos can be simultaneously unraveled via their distinctive like-sign dilepton signatures at colliders [8]. The usually considered production channel for heavy Majorana neutrinos at the LHC is $p p \rightarrow W^{ \pm} \rightarrow \ell^{ \pm} N$ (Fig. 1), with $N$ subsequently decaying to $\ell^{ \pm} W$, followed by the $W$-decay to hadronic final states. For $M_{N}>M_{W}$, the $W$-boson produced from the $p p$ collision is off-shell, whereas that coming from the $N$-decay is on-shell. For a Majorana neutrino $N$, this leads to the "smoking-gun" collider signature of same-sign dileptons plus two jets with no missing energy $\left(\ell^{ \pm} \ell^{ \pm} j j\right)$. This was first pointed out in the context of left-right models [9], and was subsequently analyzed in [10-13] within the SM seesaw.

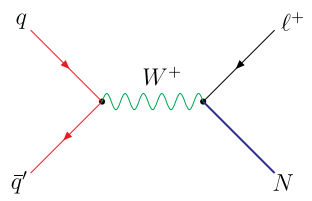

FIG. 1 (color online). The usually considered heavy neutrino production channel in the SM seesaw at the LHC. 
Experimental searches based on this channel have been performed using the $\sqrt{s}=7 \mathrm{TeV}$ LHC data for the dimuon case $[14,15]$ (and also for the di-electron case [15]). No excess above the expected SM background has been observed so far, and upper limits on the lightto-heavy neutrino mixing parameter squared, $\left|V_{\mu N}\right|^{2} \approx$ $\left(\xi \xi^{\dagger}\right)_{\mu \mu}=10^{-2}-10^{-1}$, have been derived for heavy neutrino masses $M_{N}=100-300 \mathrm{GeV}$.

For collider tests of the SM seesaw to be effective, the mixing parameter $V_{\ell N} \approx \xi_{\ell N}$ must be significant, since this is the only way the heavy neutrino communicates to the observable SM sector. This requires that apart from $M_{N}$ being small (in the sub- $\mathrm{TeV}$ to $\mathrm{TeV}$ range to be kinematically accessible), $M_{D}$ must be large (in the few $\mathrm{GeV}$ range) simultaneously. In the traditional "vanilla" seesaw mechanism, we expect the light-to-heavy neutrino mixing $V_{\ell N} \sim$ $\sqrt{m_{\nu} / M_{N}} \lesssim 10^{-7}$ for $M_{N} \sim 1 \mathrm{TeV}$, due to the smallness of light neutrino mass $m_{\nu} \lesssim 0.1 \mathrm{eV}$ [16], thus making the collider signal unobservable. However, if the Dirac and Majorana mass matrices in (2) have specific textures which can be enforced by some symmetries [10,17], $V_{\ell N}$ can be naturally large while the light neutrinos remain massless at the tree level. The observed nonzero neutrino masses and mixing can be generated by approximately breaking the underlying symmetry structure via radiative effects and/or higher-dimensional operators. Such models allow the possibility of having $\mathcal{O}(100) \mathrm{GeV}$ heavy Majorana neutrinos with a significant $V_{\ell N}$, and hence, observable lepton number violation (LNV) at the LHC [18], without being in conflict with the neutrino oscillation data. We will generically assume this for our subsequent discussion, without referring to any particular texture or model-building aspects, and so treat $M_{N}$ and $V_{\ell N}$ as free phenomenological parameters.

In this Letter we explicitly demonstrate the existence of a novel production mechanism for heavy neutrinos at the LHC which dominates over the previously considered $s$-channel $W$-exchange diagram shown in Fig. 1 . Within the SM seesaw, there exist many reactions at parton level listed in [11], which give rise to same-sign dileptons with $n \geq 2$ jets. The contributions of most of these additional diagrams are negligible compared to the that in Fig. 1, and have therefore been neglected in all previous collider analyses. As we show below, however, diagrams involving virtual photons in the $t$-channel as shown in Fig. 2 give rise to diffractive processes, such as

$$
p p \rightarrow W^{*} \gamma^{*} j j \rightarrow \ell^{ \pm} N j j
$$

which are not negligible, but infrared enhanced. In fact, the inclusive cross section of these processes is divergent due to the collinear singularity caused by the photon propagator. As we increase the virtuality of the photon by giving a large transverse momentum to the associated jet $\left(p_{T}^{j}\right)$, the cross section becomes finite. Following the
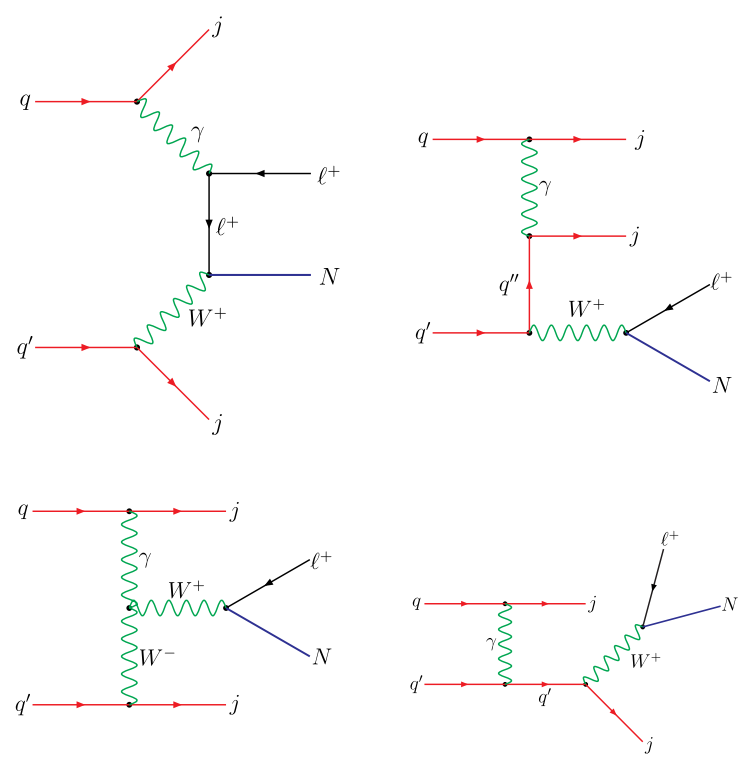

FIG. 2 (color online). New heavy neutrino production channels at the LHC. Mirror-symmetric and Z-mediated graphs are not shown.

Weizsäcker-Williams equivalent photon approximation (EPA) for electrons [19], we may analogously write down the cross section as a convolution of the probability that the proton radiates off a real photon, by absorbing the collinear divergence of the low- $p_{T}^{J}$ regime into an effective photon structure function for the proton [20,21].

To establish the importance of the diagrams in Fig. 2, we compare the inclusive cross section for $N \ell^{ \pm} j j$ with the previously considered $N \ell^{ \pm}$in Fig. 1 . Note that the $p p \rightarrow N \ell^{ \pm} j j$ process receives contributions from both hadronic and electroweak processes. The hadronic channels mediated by virtual gluons and quarks give $\mathcal{O}\left(\alpha_{s}\right)$ corrections to the production channel in Fig. 1 and drop at the same rate as the $p p \rightarrow N \ell^{ \pm}$cross section, as the heavy neutrino mass increases. The electroweak contributions come from the virtual $\gamma$-exchange diagrams shown in Fig. 2, and also from additional $W^{ \pm} Z$-mediated graphs not shown here. All these Feynman graphs must be taken into account, in order to get a gauge-invariant result. It turns out that the total electroweak contribution drops at a rate slower than the $p p \rightarrow N \ell^{ \pm}$cross section with increasing heavy neutrino mass. This is mainly due to the infraredenhanced cross section of the $\gamma$-mediated processes in (3), which have a significantly milder dependence on $M_{N}$. As a result, the production channel (3) dominates over the earlier considered $p p \rightarrow N \ell^{ \pm}$channel with increasing $M_{N}$. Similar behavior is also expected with increasing center of mass energy $\sqrt{s}$ in the $p p$ collisions, as verified by our numerical simulations given below. Thus, the process (3) becomes increasingly important for heavy neutrino searches at the LHC, for higher energies $\sqrt{s}$ and also larger $M_{N}$ values. Consequently, it must be taken into account in present and future analyses of the LHC data. 

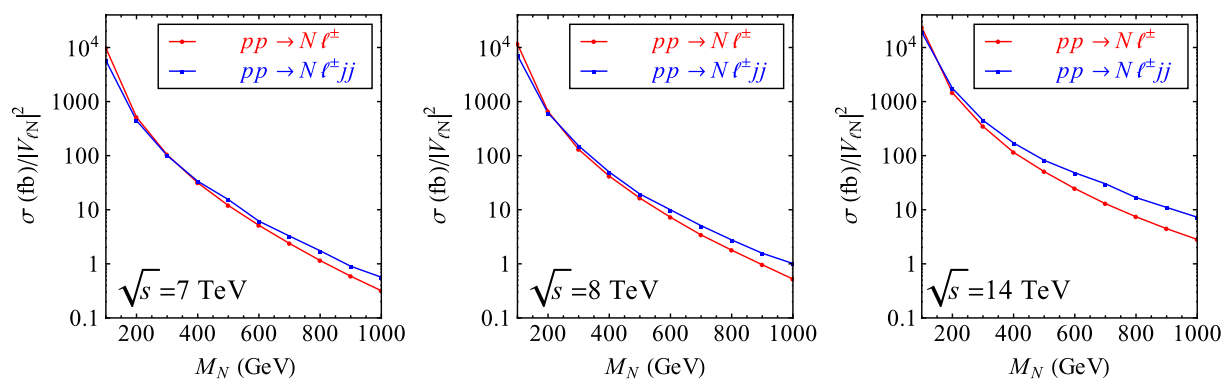

FIG. 3 (color online). Comparison of the inclusive cross sections for the heavy neutrino production channels $p p \rightarrow N \ell^{ \pm}$and $p p \rightarrow N \ell^{ \pm} j j$ at LHC energies of $\sqrt{s}=7,8$ and $14 \mathrm{TeV}$.

Our numerical results are shown in Fig. 3 for the inclusive production cross sections normalized to the mixing parameter $\left|V_{\ell N}\right|^{2}=1$. For the process $p p \rightarrow N \ell^{ \pm} j j$, we obtain the "inclusive" cross section by applying a minimal jet $p_{T}$ cut of $p_{T}^{j}>\left(p_{T}^{j}\right)_{\min }$ to avoid the collinear singularity, whereas the infrared part is approximated by the inclusive cross section of the process $p \gamma \rightarrow N \ell^{ \pm} j$, where the photon comes from a proton. The latter process was calculated with EPA using the improved Weizsäcker-Williams formula [20] for a fixed factorization scale of $\mu_{F}=\left(p_{T}^{j}\right)_{\min }$. For concreteness, we have chosen $\left(p_{T}^{j}\right)_{\min }=10 \mathrm{GeV}$ (the lowest detection threshold for ATLAS) and used the equivalent photon distribution functions as implemented in MADGRAPH5 [22], whereas the quark and gluon distribution functions of the proton were taken from CTEQ6L [23]. The renormalization scale was chosen for each event depending on the maximum final-state mass $\left(M_{N}\right.$ in our case). Note that the total cross section which is a sum of the $p p \rightarrow N \ell^{ \pm} j j$ and $p \gamma \rightarrow N \ell^{ \pm} j$ cross sections should be independent of the $p_{T}^{j}$ cut, as long as the collinear part of the $p p \rightarrow N \ell^{ \pm} j j$ process is consistently absorbed into the photon distribution function. We observed some discrepancy from this general expectation, which could be due to the fact that the accuracy of EPA, while being excellent for elastic scattering processes [24], is scaledependent for inelastic channels [25], and moreover, the choice of the factorization scale is not unique due to higher order effects in perturbative QCD. For an alternative model of EPA as currently implemented in CALCHEP3.4 [26], we get similar results as above for the $p \gamma \rightarrow N \ell^{ \pm} j$ cross sections within an accuracy of $10 \%-20 \%$. However, since the dominant contribution results from the process $p p \rightarrow$ $N \ell^{ \pm} j j$ in the current mass range of interest, this subtle point will not change the main results of the analysis presented here.

From Fig. 3 we see that the $N \ell^{ \pm}$production channel is dominant only in the low mass regime, whilst the new $N \ell^{ \pm} j j$ channel starts becoming dominant for $M_{N} \gtrsim$ $300 \mathrm{GeV}$ at $\sqrt{s}=7 \mathrm{TeV}$ LHC. This crossover point shifts towards lower values of $M_{N}$, with increasing $\sqrt{s}$. It is interesting to note that the existing heavy neutrino searches $[14,15]$ have only explored up to $M_{N}=300 \mathrm{GeV}$ with $\sqrt{s}=7 \mathrm{TeV}$ LHC data, but plan to extend up to $M_{N}=$ $500 \mathrm{GeV}$ with $\sqrt{s}=8 \mathrm{TeV}$ data. Hence, the new production channel proposed here must be taken into account in all current and future LHC analyses.

An important consequence of the new production mechanism for heavy neutrinos is that the current LHC sensitivity for the light-to-heavy neutrino mixing parameter $V_{\mu N}$ can be improved significantly for the whole heavy neutrino mass range of interest, i.e., $M_{N}=100-300 \mathrm{GeV}$. In order to derive the new limits on $V_{\mu N}$, we first calculate the efficiency of the new signal proposed here: $p p \rightarrow$ $N \mu^{ \pm} j j \rightarrow \mu^{ \pm} \mu^{ \pm} 4 j$, after implementing the same selection criteria as used for the $\mu^{ \pm} \mu^{ \pm} j j$ channel in the $\sqrt{s}=7 \mathrm{TeV}$ ATLAS analysis [14],

$$
\begin{aligned}
p_{T}^{j} & >20 \mathrm{GeV}, \quad p_{T}^{\mu}>20 \mathrm{GeV}, \quad p_{T}^{\mu, \text { leading }}>25 \mathrm{GeV}, \\
\left|\eta^{j}\right| & <2.8, \quad\left|\eta^{\mu}\right|<2.5, \quad \Delta R^{j j}>0.4, \quad \Delta R^{\mu j}>0.4, \\
m_{\mu \mu} & >15 \mathrm{GeV}, \quad E_{T}^{\text {miss }}<35 \mathrm{GeV}, \quad m_{j j} \in[55,120] \mathrm{GeV},
\end{aligned}
$$

and for $\Delta R^{\mu j}<0.4$, we require $p_{T}^{\mu}>80 \mathrm{GeV}$ to retain muons close to jets from event topologies with boosted heavy neutrinos. After generating the parton level events with MADGRAPH5 [22], the showering and hadronization were performed with Pythia6.4 [27] and a fast detector simulation was done using DelPHES2.0.5 [28]. Jets are reconstructed using the anti- $k_{T}$ jet clustering algorithm with $R=0.4$ as implemented in FASTJET2 [29]. We find that the total selection efficiency for the $\mu^{ \pm} \mu^{ \pm}$signal remains almost the same as before [14], since the additional two jets coming from the new channel are usually lost due to the stringent selection criteria given in (4). Regarding the SM background for these processes, we expect the background for di-muon $+n$ jets (with $n \geq 2$ ) to be the same as that reported in [14] for the selection criteria in (4). Note that the SM backgrounds for the $\mu^{ \pm} \mu^{ \pm} 4 j$ signal reported here mainly come from $t \bar{t}+V$ (where $V=W, Z$ ) and $W W$ production, which are small compared to the $W Z$ background for the $\mu^{ \pm} \mu^{ \pm} j j$ signal [14]. A separate dedicated set of selection criteria and background reduction methods must be designed in order to distinguish the new $\mu^{ \pm} \mu^{ \pm} 4 j$ 


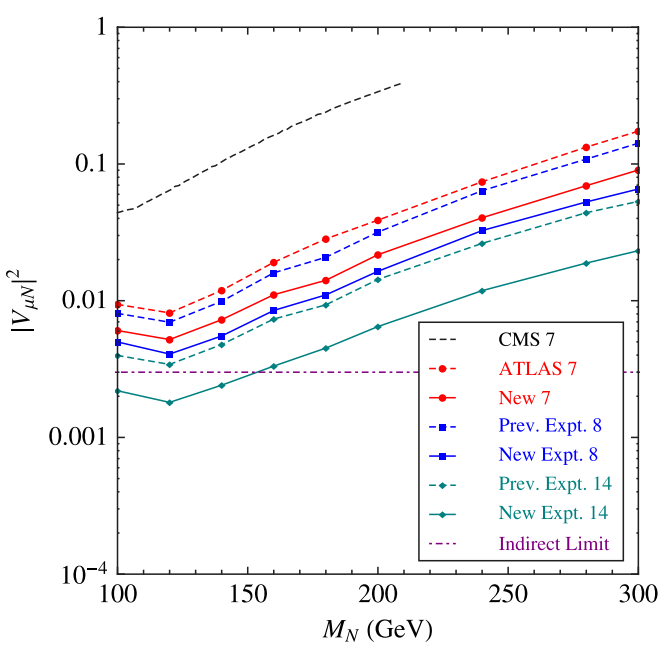

FIG. 4 (color online). Improved upper limits (solid lines, also labeled "New") on the mixing parameter $\left|V_{\mu N}\right|^{2}$ for LHC energies $\sqrt{s}=7,8$ and $14 \mathrm{TeV}$, along with the current CMS [15] and ATLAS [14] limits derived at $\sqrt{s}=7 \mathrm{TeV}$, and the conservative upper limits expected for 8 - and $14-\mathrm{TeV}$ LHC runs using the previous production mode of Fig. 1 (dashed lines, also labeled "Prev."). The horizontal line shows the current best limit on $\left|V_{\mu N}\right|^{2}$ from indirect searches [30].

signal proposed in this Letter from the usual $\mu^{ \pm} \mu^{ \pm} j j$ signal, and this will be studied elsewhere. A similar analysis can also be performed for the di-electron signal $e^{ \pm} e^{ \pm} n j$ (with $n \geq 2$ ). Although the limits on $\left|V_{e N}\right|^{2}$ derived from $0 \nu \beta \beta$ constraints are much more stringent [8], models with quasidegenerate heavy Majorana neutrinos may naturally evade these constraints, while giving rise to sizable LNV signals at the LHC [13]. For the corresponding limits on $\left|V_{\tau N}\right|^{2}$, the identification of same-sign di-tau events at the LHC is quite difficult, thus making a realistic collider simulation for this case rather involved.

Following a rather conservative approach to our analysis here, we use the current $95 \%$ confidence level upper limits on the cross section $\sigma\left(p p \rightarrow \mu^{ \pm} \mu^{ \pm} j j\right)$ [14], derived from the $\sqrt{s}=7 \mathrm{TeV}$ LHC data with $4.7 \mathrm{fb}^{-1}$ luminosity, and translate them into upper limits on the mixing parameter $\left|V_{\mu N}\right|^{2}$ as shown in Fig. 4 by dividing the cross section limits by the total inclusive cross section $\sigma\left(p p \rightarrow \mu^{ \pm} \mu^{ \pm} n j\right)$ (with $n \geq 2$ ). We find that the existing ATLAS limits [14] are improved by almost $50 \%$ with the inclusion of the new production mechanism. For comparison, we also show the corresponding CMS limits [15] which are much weaker compared to those by ATLAS, mainly due to their large backgrounds. The horizontal line shows the current best limit on $\left|V_{\mu N}\right|^{2}$ derived indirectly from electroweak precision data [30] which is independent of the heavy neutrino mass for $M_{N}>M_{Z}$. Note that the LFV processes (such as rare lepton decays [31] and $\mu-e$ conversion [32]) put stringent constraints on the product $\left|V_{\ell N} V_{\ell^{\prime} N}^{*}\right|$ (with $\ell \neq \ell^{\prime}$ ) [6], thereby limiting the LHC sensitivity for LFV signals of the type $e^{ \pm} \mu^{ \pm} j j$; however, they do not restrict the individual mixing parameters $\left|V_{\ell N}\right|^{2}$ in a generic low-scale seesaw model. In order to compare the direct search limits with the indirect one, we also derive our expected upper limits for $\sqrt{s}=8$ and $14 \mathrm{TeV}$ LHC by assuming that the corresponding experimental upper limits on the signal cross section will be at least as good as the $\sqrt{s}=7 \mathrm{TeV}$ results. Again, these are conservative limits as the experimental limits on cross section are expected to improve significantly with the analysis of more data, if no signal is observed. In that case, the direct collider limits could surpass the indirect limits for a significant range of heavy neutrino masses, once the new production mechanism proposed here is considered. In particular, Fig. 4 shows that the effect of the new production mechanism at LHC energies $\sqrt{s}=14 \mathrm{TeV}$ will be to improve the current ATLAS limit by at least a factor of five.

In summary, we have analyzed a new dominant production mechanism for heavy neutrinos at the LHC. This mechanism is extremely important for the range of heavy neutrino masses currently being searched for and provides significantly improved direct limits on the light-to-heavy neutrino mixing $V_{\ell N}$, in a fully independent fashion of the indirect searches. As more data are gathered at the LHC and the sensitivity to higher heavy neutrino mass ranges is contemplated, these new contributions will be crucial in setting the best possible direct limits on the mixing parameter $V_{\mu N}$ in the absence of a signal. On the other hand, an evidence of LNV at the LHC could reveal underlying symmetries of the lepton sector, thus shedding light on the seesaw mechanism. We should note that the scope of the new infrared-enhanced production mechanism proposed here is not just limited to heavy Majorana neutrinos, and can also be applied to other heavy particle searches (the so-called "exotics") at the LHC. For instance, for pseudo-Dirac heavy neutrinos, the same production channels studied here could give rise to an enhanced trilepton signal. This mechanism is also applicable for searches of vectorlike fermions and new charged scalars. We hope to address some of these aspects in a future communication.

P. S. B. D. and A. P. thank Mike Seymour for helpful discussions on the equivalent photon approximation. P. S. B. D. also thanks Francisco del Aguila and Rabindra Mohapatra for useful discussions on lepton number violation, Olivier Mattelaer for help with photon structure functions in MADGRAPH, and John Almond and Joel Klinger for clarifications on the ATLAS selection cuts used here. The work of P. S. B. D. and A. P. is supported by the Lancaster-Manchester-Sheffield Consortium for Fundamental Physics under STFC Grant No. ST/ J000418/1. In addition, A.P. gratefully acknowledges partial support by a IPPP associateship from Durham University. The work of U.K.Y. is supported by the NSF of Korea under Grant No. 2013R12A2A2A01069162. 
[1] For a review, see R. N. Mohapatra, and A. Y. Smirnov, Annu. Rev. Nucl. Part. Sci. 56, 569 (2006).

[2] P. Minkowski, Phys. Lett. 67B, 421 (1977); T. Yanagida, in Proceedings of the Workshop on Unified Theories and Baryon Number in the Universe, edited by A. Sawada and A. Sugamoto (KEK, Tsukuba, 1979); M. Gell-Mann, P. Ramond, and R. Slansky, in Supergravity, edited by P. Van Niewenhuizen (D. Freeman, North Holland, Amsterdam, 1980); R. N. Mohapatra and G. Senjanović, Phys. Rev. Lett. 44, 912 (1980).

[3] J. Schechter and J. W. F. Valle, Phys. Rev. D 22, 2227 (1980); 25, 774 (1982).

[4] For reviews, see W. Rodejohann, Int. J. Mod. Phys. E 20, 1833 (2011); F. F. Deppisch, M. Hirsch, and H. Pas, J. Phys. G 39, 124007 (2012).

[5] S. Antusch, C. Biggio, E. Fernandez-Martinez, M. B. Gavela, and J. Lopez-Pavon, J. High Energy Phys. 10 (2006) 084; A. Abada, C. Biggio, F. Bonnet, M. B. Gavela, and T. Hambye, J. High Energy Phys. 12 (2007) 061.

[6] For a review, see M. Raidal et al., Eur. Phys. J. C 57, 13 (2008).

[7] M. Malinsky, T. Ohlsson and H. Zhang, Phys. Rev. D 79, 073009 (2009); M. Malinsky, T. Ohlsson, Z. -z. Xing, and H. Zhang, Phys. Lett. B 679, 242 (2009); P. S. Bhupal Dev and R. N. Mohapatra, Phys. Rev. D 81, 013001 (2010); D. V. Forero, S. Morisi, M. Tortola, and J. W. F. Valle, J. High Energy Phys. 09 (2011) 142.

[8] For a review, see A. Atre, T. Han, S. Pascoli, and B. Zhang, J. High Energy Phys. 05 (2009) 030.

[9] W.-Y. Keung and G. Senjanović, Phys. Rev. Lett. 50, 1427 (1983).

[10] A. Pilaftsis, Z. Phys. C 55, 275 (1992).

[11] A. Datta, M. Guchait and A. Pilaftsis, Phys. Rev. D 50, 3195 (1994).

[12] F. M. L. Almeida, Jr, Y. A. Coutinho, J. A. Martins Simoes, and M. A. B. do Vale, Phys. Rev. D 62, 075004 (2000); O. Panella, M. Cannoni, C. Carimalo, and Y. N. Srivastava, Phys. Rev. D 65, 035005 (2002); T. Han and B. Zhang, Phys. Rev. Lett. 97, 171804 (2006); F. del Aguila, J. A. Aguilar-Saavedra, and R. Pittau, J. High Energy Phys. 10 (2007) 047; F. del Aguila and J. A. Aguilar-Saavedra, Nucl. Phys. B813, 22 (2009).

[13] S. Bray, J. S. Lee and A. Pilaftsis, Nucl. Phys. B786, 95 (2007).

[14] ATLAS Collaboration, Report No. ATLAS-CONF-2012139.

[15] S. Chatrchyan et al. (CMS Collaboration), Phys. Lett. B 717, 109 (2012).
[16] P. A. R. Ade et al. (Planck Collaboration), arXiv: 1303.5076.

[17] J. Gluza, Acta Phys. Pol. B 33, 1735 (2002); A. Pilaftsis, Phys. Rev. Lett. 95, 081602 (2005); J. Kersten and A. Y. Smirnov, Phys. Rev. D 76, 073005 (2007); Z.-z. Xing, Prog. Theor. Phys. Suppl. 180, 112 (2009); X.-G. He, S. Oh, J. Tandean, and C. -C. Wen, Phys. Rev. D 80, 073012 (2009); A. Ibarra, E. Molinaro, and S. T. Petcov, J. High Energy Phys. 09 (2010) 108; F. F. Deppisch, and A. Pilaftsis, Phys. Rev. D 83, 076007 (2011); C. H. Lee, P. S. Bhupal Dev, and R. N. Mohapatra, Phys. Rev. D 88, 093010 (2013).

[18] Even for quasidegenerate heavy Majorana neutrinos (as occurs in specific models), the LNV signal can be sizable when the mass splitting $\Delta M_{N} \equiv M_{N_{1}}-M_{N_{2}}$ is comparable to the width $\Gamma_{N} \equiv\left(\Gamma_{N_{1}}+\Gamma_{N_{2}}\right) / 2$ [13]. For instance, for the on-shell production of $N_{1,2}$ with average fourmomentum squared $\bar{s}=\left(M_{N_{1}}^{2}+M_{N_{2}}^{2}\right) / 2$, the LNV amplitude in the muon-channel is given by $\mathcal{A}_{\mathrm{LNV}}^{\mu \mu}(\bar{s})=-V_{\mu N_{1}}^{2}\left(2 \Delta M_{N} / \Delta M_{N}^{2}+\Gamma_{N}^{2}\right)+\mathcal{O}\left(\Delta M_{N} / M_{N_{1}}\right)$, for $\Delta M_{N} \lesssim \Gamma_{N}$.

[19] C. F. von Weizsäcker, Z. Phys. 88, 612 (1934); E. J. Williams, Phys. Rev. 45, 729 (1934).

[20] V. M. Budnev, I. F. Ginzburg, G. V. Meledin, and V. G. Serbo, Phys. Rep. 15, 181 (1975).

[21] S. Frixione, M. L. Mangano, P. Nason, and G. Ridolfi, Phys. Lett. B 319, 339 (1993); M. Glück, C. Pisano and E. Reya, Phys. Lett. B 540, 75 (2002).

[22] J. Alwall, M. Herquet, F. Maltoni, O. Mattelaer and T. Stelzer, J. High Energy Phys. 06 (2011) 128.

[23] J.Pumplin, D. R. Stump, J. Huston, H. L. Lai, P. M. Nadolsky, and W. K. Tung, J. High Energy Phys. 07 (2002) 012.

[24] B. A. Kniehl, Phys. Lett. B 254, 267 (1991).

[25] C. Pisano, Eur. Phys. J. C 38, 79 (2004).

[26] A. Belyaev, N. D. Christensen, and A. Pukhov, Comput. Phys. Commun. 184, 1729 (2013).

[27] T. Sjostrand, S. Mrenna, and P. Z. Skands, J. High Energy Phys. 05 (2006) 026.

[28] S. Ovyn, X. Rouby, and V. Lemaitre, arXiv:0903.2225; J. de Favereau et al, arXiv:1307.6346.

[29] M. Cacciari, G. P. Salam, and G. Soyez, Eur. Phys. J. C 72, 1896 (2012).

[30] F. del Aguila, J. de Blas, and M. Perez-Victoria, Phys. Rev. D 78, 013010 (2008).

[31] A. Ilakovac and A. Pilaftsis, Nucl. Phys. B437, 491 (1995).

[32] R. Alonso, M. Dhen, M. B. Gavela, and T. Hambye, J. High Energy Phys. 01 (2013) 118. 